\title{
QUANTUM CHEMICAL ANALYSIS OF URANIUM TRIOXIDE CONFORMERS
}

\author{
M. B. Shundalau ${ }^{a^{*}}$ and D. S. Umreiko
}

UDC 539.19

The shapes of two hypothetical conformers of uranium trioxide $\mathrm{UO}_{3}$ were analyzed by DFT calculations and the structures of localized molecular orbitals (LMOS). It was shown that differences between the Y-and T-shapes of UO 3 were due mainly to the different contributions of the $U 6 p_{z}$-and $6 p_{x}$-orbitals to the corresponding LMOs and to the formation of specifically shaped regions of higher electron density in the vicinity of this atom.

Keywords: ab initio calculation, density functional theory, effective core potential, uranium trioxide, localized molecular orbitals.

Introduction. The structural and physicochemical characteristics of molecular systems can be reproduced to a high degree of accuracy by modern quantum chemical methods. This makes it possible to use quantum chemical modeling to make predictions. A textbook example of this is the prediction based on ab initio calculations and then the experimental confirmation of the existence of hypervalent molecules of the $\mathrm{CLi}_{5}$ type [1-4]. Actinides, in particular uranium, are traditionally treated from a quantum chemical viewpoint as rather complicated atoms because factors that are immaterial for compounds containing light atoms must be considered in order to obtain an adequate reproduction and/or prediction of the electronic structure and other characteristics of molecular systems involving them. These include the significant number of active orbitals of partially filled $d$ - and $f$-subshells, the consideration of relativistic corrections for core electrons, substantial correlation effects, etc. Quantum chemical methods in combination with various experimental methods enabled a large number of simple hydrides, oxides, nitroxides, and carboxides of uranium and other actinides to be identified and traditional U-containing compounds (e.g., uranyl ion and its complexes) to be analyzed [5]. Theoretical investigations of molecular systems containing the $\mathrm{U}$ atom that have not yet been confirmed experimentally include the quantum chemical prediction of a stable U-U bond [6].

Uranium trioxide $\left(\mathrm{UO}_{3}\right)$ is a $\mathrm{U}(\mathrm{VI})$ compound with at least six crystalline polymorphic modifications. The trioxide in the molecular form in addition to other very simple $\mathrm{U}$ oxides $\left(\mathrm{UO}, \mathrm{UO}_{2}\right)$ and several of their ions were studied in lowtemperature matrix isolation [7-11]. Vibrational IR spectra of $\mathrm{UO}_{3}$ were analyzed based on the assumption that this molecule isolated in a low-temperature matrix had a structure close to T-shaped. It is noteworthy that gas-phase experimental data on the $\mathrm{UO}_{3}$ structure have not been reported.

The previous assumptions [7-11] were confirmed by high-level modern quantum chemical calculations [12]. Because electrons of $6 s-, 6 p-, 7 s-, 7 p-, 6 d$-, and $5 f$-subshells are involved in the formation of chemical bonds to $\mathrm{U}$ [13], there is a basis to suggest that the direction of its chemical bonds depends to a large extent on the type of hybridization of these atomic orbitals (AOs). This can lead to the existence of various stable isomeric shapes of $\mathrm{U}(\mathrm{VI})$ molecular compounds.

We found earlier based on an LANL2DZ/B3LYP/cc-pVDZ quantum chemical calculation [14] that $\mathrm{UO}_{3}$ in the molecular form has two equilibrium configurations. In addition to a minimum corresponding to the T-shape of $\mathrm{UO}_{3}\left(156.3^{\circ}\right.$ angle between $\mathrm{O}(1)=\mathrm{U}=\mathrm{O}(2)$ uranyl bonds), the potential curve had another minimum lying $216 \mathrm{~cm}^{-1}$ below the T-shape and corresponding to the Y-shape $\left(88.7^{\circ}\right.$ angle between uranyl bonds). Figure 1 shows the T- and Y-shapes of $\mathrm{UO}_{3}$. Both configurations have $C_{2 v}$ point symmetry. The potential barrier separating the configurations corresponds to a $120^{\circ}$ angle and has a height of $2731 \mathrm{~cm}^{-1}$. Calculations were performed for frozen $\mathrm{O}(1) \mathrm{UO}(3)$ and $\mathrm{O}(2) \mathrm{UO}(3)$ angles. The geometry was optimized over the remaining internal coordinates with an initial angle $\mathrm{O}(1)=\mathrm{U}=\mathrm{O}(2) \sim 90^{\circ}$ for the Y-shape and $\sim 180^{\circ}$ for the T-shape. The lack in the calculated vibrational spectra of imaginary frequencies confirmed that both configurations were stable in terms of this approximation.

\footnotetext{
${ }^{*}$ To whom correspondence should be addressed.
}

${ }^{\mathrm{a} B e l a r u s i a n}$ State University, 4 Nezavisimost' Ave., 220030, Minsk, Belarus; e-mail: shundalov@bsu.by; ${ }^{b}$ A. N. Sevchenko Institute of Applied Physical Problems, Belarusian State University, Minsk; e-mail: lab_dozator@mail.ru. Translated from Zhurnal Prikladnoi Spektroskopii, Vol. 80, No. 6, pp. 817-822, November-December, 2013. Original article submitted June 10, 2013. 

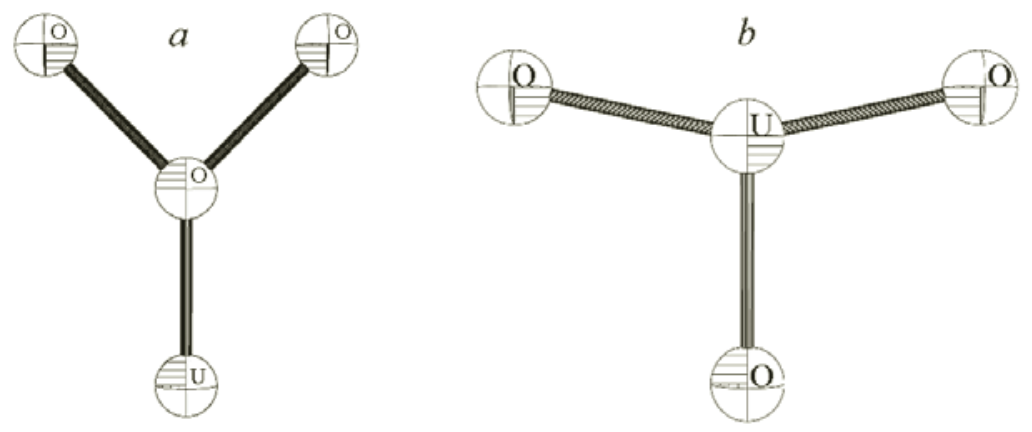

Fig. 1. Equilibrium configurations of $\mathrm{UO}_{3}$ : Y-shape (a) and T-shape (b).

Without disregarding completely the possibility that the two equilibrium configurations of $\mathrm{UO}_{3}$ could be an artefact due to assumptions used in the calculation, we postulated that the existence of such stable shapes did not contradict quantum mechanical concepts about the structure of molecular compounds and the formation of chemical bonds and could be explained in terms of localized molecular orbitals (LMOs). It is well known that LMOs can correspond to atomic cores, chemical bonds, or unshared electron pairs [15]. If the shape of the LMOs reflects the type and direction of the chemical bond, then an analysis of the contributions of various AOs to a given LMO and a comparison of analogs of LMOs for different compounds (or conformers of a single compound) of similar electronic structures enables the specifics of their steric structures to be determined.

Calculation Method. Equilibrium structures were optimized and force fields and vibrational eigen frequencies were calculated in the harmonic approximation for $\mathrm{UO}_{3}$ conformers and $\mathrm{UO}_{2}{ }^{2+}$ using the applied quantum chemical program GAMESS-US [16, 17]. The MacMolPlt [18] and ORTEP [19] programs were used to visualize the obtained results. The relativistic effective core potential (RECP) LANL2DZ [20] was used to approximate the $U$ atom by replacing 78 inner electrons (Large Core approximation); specially developed DZ basis sets for this RECP, for the other U electrons. The O atoms were described based on standard full-electron correlation-consistent Dunning basis families cc-pVnZ and cc-pCVnZ [21]. The RECP and corresponding basis sets were generated using the Extensible Computational Chemistry Environment Basis Set Database [22-24]. The hybrid exchange-correlation functional B3LYP was also used in all calculations [25-27]. The approximation used in the calculations was also successful for modeling the structure and vibrational spectra of U-containing compounds $\left(\mathrm{UO}_{2} \mathrm{Cl}_{2}, \mathrm{UCl}_{4}\right.$, and their complexes) $[28,29]$.

LMOs were constructed by the Edmiston and Ruedenberg method [30]. Two types of designations were used for spherical functions with a non-zero $l$ value, i.e., with Cartesian projections or with an $\left|m_{l}\right|$ value. The signs "+" or "-" in this instance corresponded to the combination sum or difference of functions with $m_{l}$ with opposite signs. A general set was used for the $f$-orbitals. Thus, alternative designations $p_{0}, p_{+1}$, and $p_{-1}$ corresponded to the set of spherical functions $p_{z}, p_{x}$, and $p_{y}$; $d_{0}, d_{+1}, d_{-1}, d_{+2}, d_{-2}$, to the set $d_{2 x^{2}-x^{2}-y^{2}}, d_{x z}, d_{y z}, d_{x^{2}-y^{2}}, d_{x y} ; f_{0}, f_{+1}, f_{-1}, f_{+2}, f_{-2}, f_{+3}, f_{-3}$, to the set $f_{2 z^{3}-3 z\left(x^{2}+y^{2}\right)}\left(\right.$ or $\left.f_{z 3}\right)$, $f_{x\left(4 z^{2}-x^{2}-y^{2}\right)}$ (or $\left.f_{x z^{2}}\right), f_{y\left(4 z^{2}-x^{2}-y^{2}\right)}$ (or $\left.f_{y z^{2}}\right), f_{z\left(x^{2}-y^{2}\right)}, f_{x y z}, f_{x\left(x^{2}-3 y^{2}\right)}, f_{y\left(3 x^{2}-y^{2}\right)}$. In each instance, the designations were chosen due to convenience and appearance.

Results and Discussion. $\mathrm{UO}_{3}$ can be viewed as the simplest example of equatorial coordination of $\mathrm{O}$ to $\mathrm{UO}_{2}{ }^{2+}$ [31]. This approach is reflected in one of the names for $\mathrm{UO}_{3}$, i.e., uranyl oxide. Therefore, structural features of the $\mathrm{UO}_{3}$ conformers can be determined by relying on concepts about the uranyl electronic structure, which was studied several times by quantum chemistry at various theory levels $[13,32-38]$. It was shown that uranyl $\mathrm{U}=\mathrm{O}$ bonds have $\sigma$ - and $\pi$-type features. The $\sigma$-type orbitals are formed by overlap of $6 d_{0}$ - and $7 s$-orbitals (symmetric MO) or $5 f_{0^{-}}$and $6 p_{0}$-orbitals (asymmetric MO) of $\mathrm{U}$ with hybrid $s p_{z}$-orbitals of $\mathrm{O}$ atoms. The $\pi$-type MOs are formed by overlap of $6 d_{x z^{-}}$and $6 d_{y z}$-orbitals (symmetric MO) or $6 p_{x^{-}}, 6 p_{y^{-}}, 5 f_{x z^{2}}$, and $5 f_{y z^{2}}$-orbitals (asymmetric MO) of $\mathrm{U}$ with $\mathrm{O} 2 p_{x^{-}}$and $2 p_{y^{-}}$-orbitals. The $\pi$-electrons are localized near the $\mathrm{O}$ atoms so that the order of the uranyl bonds is intermediate between a value of 2 and 3 .

The active space for uranyl ion in the Large Core RECP approximation consists of 28 electrons that form 14 LMOs. Three of them $\left(U_{\mathrm{I}}, U_{\mathrm{II}}\right.$, and $\left.U_{\mathrm{III}}\right)$ correspond to $1 s$-orbitals of $\mathrm{O}$ atoms and $6 s$-orbitals of the $\mathrm{U}$ atom; another two $\left(U_{\mathrm{V}}\right.$ and $\left.U_{\mathrm{VI}}\right)$, to $\mathrm{U} 6 p_{x}$ - and $6 p_{y}$-orbitals. These LMOs are localized on the atoms, i.e., do not correspond to chemical bonds. They will not be further examined. The remaining 9 LMOs are divided into three groups. The first of these consists of LMO $U_{\mathrm{IV}}$; the second, 6 LMOs ( $\left.U_{\mathrm{VII}}, U_{\mathrm{VIII}}, U_{\mathrm{IX}}, U_{\mathrm{X}}, U_{\mathrm{XII}}, U_{\mathrm{XIII}}\right)$; the third, $2 \mathrm{LMOs}\left(U_{\mathrm{XI}}, U_{\mathrm{XIV}}\right)$. 
The $U_{\text {IV }}$ LMO (Fig. $\left.2 \mathrm{a}\right)$ is formed from $\mathrm{U} 6 p_{0^{-}}(66 \%)$ and $5 f_{0^{-}}$orbitals $(1 \%)$ and $\mathrm{O} s-(28 \%)$ and $p_{0^{-}}$-orbitals $(5 \%)$ (here and henceforth the separate AOs are rounded to units of \%) and is a $\sigma$-orbital. Each LMO of the pair $U_{\mathrm{XI}}$ and $U_{\mathrm{XIV}}$ (Fig. 2c) is localized on one of the uranyl bonds and is formed from $U 6 s-(3 \%), 7 s-(2 \%), 6 p_{0^{-}}(21 \%), 6 d_{0^{-}}(7 \%), 5 f_{0^{-}}$orbitals $(28 \%)$ and $s-(7 \%)$ and $p_{0}$-orbitals (32\%) of the corresponding $\mathrm{O}$ atom and is also a $\sigma$-type orbital. The six remaining LMOs are divided into triplets ( $U_{\mathrm{VII}}, U_{\mathrm{XII}}, U_{\mathrm{XIII}}$ and $U_{\mathrm{VIII}}, U_{\mathrm{IX}}, U_{\mathrm{X}}$ ) localized on each of the uranyl bonds (Fig. 2b). The LMOs of each triplet are rotated relative to each other around the $z$ axis by $120^{\circ}$. All aforementioned types of active subshells of $\mathrm{U}$ with $m_{l}=0, \pm 1$ in addition to $s$ - $(26 \%)$ and $p_{0}$-orbitals $(59 \%)$ of the corresponding $\mathrm{O}$ atom contribute to these LMOs. Of the U AOs, the U $6 p_{x^{-}}(1 \%), 6 p_{y^{-}}(1 \%), 6 p_{z^{-}}(8 \%), 6 d_{+1^{-}}(2 \%), 6 d_{-1^{-}}(1 \%)$, and $5 f_{+1^{-}}$orbitals $(1 \%)$ contribute most to the $U_{\mathrm{VII}^{-}}$orbital. LMOs of this type are $\pi$-orbitals. In general, uranyl LMOs are in agreement with concepts about the electronic structure of this compound [13, 32-38]. The calculated order of the uranyl bonds is 2.38 .

Let us examine the $\mathrm{T}$ - and Y-shapes of $\mathrm{UO}_{3}$. The active space for $\mathrm{UO}_{3}$ in the approximation given above is limited to 38 electrons that form 19 LMOs. The following correspondence occurs between the Cartesian systems for the trioxide and uranyl: $x, y, z$ (trioxide) $\leftrightarrow z, x, y$ (uranyl). Therefore, for example, the analog of the $p_{0^{-}}\left(p_{z^{-}}\right)$orbital for the uranyl $\mathrm{U}$ atom is the $p_{+1^{-}}\left(p_{x^{-}}\right)$orbital of the trioxide $\mathrm{U}$ atom. For the T-shape of the trioxide, the LMOs $T_{\mathrm{I}}, T_{\mathrm{II}}, T_{\mathrm{IV}}, T_{\mathrm{VIII}}$, and $T_{\mathrm{XV}}$ are analogs of $U_{\mathrm{I}}, U_{\mathrm{II}}, U_{\mathrm{III}}, U_{\mathrm{V}}$, and $U_{\mathrm{VI}} \mathrm{LMOs}$ of uranyl, respectively, with $T_{\mathrm{III}}$ localized on the third $\mathrm{O}$ atom and $T_{\mathrm{VII}}$, on all three $\mathrm{O}$ atoms. These LMOs are not examined further. The remaining $12 \mathrm{LMOs}$ can be separated into five groups: one LMO $\left(T_{\mathrm{V}}\right)$, one LMO ( $\left.T_{\mathrm{VI}}\right)$, six LMOs ( $\left.T_{\mathrm{X}}, T_{\mathrm{XI}}, T_{\mathrm{XII}}, T_{\mathrm{XIII}}, T_{\mathrm{XIV}}, T_{\mathrm{XVI}}\right)$, three LMOs ( $\left.T_{\mathrm{IX}}, T_{\mathrm{XVII}}, T_{\mathrm{XVIII}}\right)$, and one LMO ( $\left.T_{\mathrm{XIX}}\right)$

The $T_{\mathrm{V}} \mathrm{LMO}$ (Fig. $3 \mathrm{a}$ ) is an analog of $U_{\mathrm{IV}}$. It is formed from $\mathrm{U} 6 p_{x}$-orbitals $(62 \%)$ in addition to $s-(33 \%)$ and $p_{x}$ orbitals $(4 \%)$ of uranyl $\mathrm{O}$ atoms. The total contribution of $\mathrm{U}$ and $\mathrm{O}$ orbitals to non-zero $z$-projections $\left(d_{x z}, f_{x z 2}\right.$, etc.) is $\sim 1 \%$. The $T_{\mathrm{VI}} \mathrm{LMO}$ has no analog among the uranyl LMOs. It is localized mainly on the bond to the third $\mathrm{O}$ atom and has a clearly pronounced section of increased electron density (lobe) between the uranyl bonds (Fig. 3b). The shape of this $\sigma$-type LMO is due to the dominant contribution of the $\mathrm{U} 6 p_{z}$-orbital (67\%) in addition to $2 s$ - and $2 p_{z}$-orbitals of the third $\mathrm{O}$ atom (25\%). The contributions of the uranyl $\mathrm{O} s$ - and $p_{z}$-orbitals to this LMO are $\sim 7 \%$. The next six LMOs $\left(T_{\mathrm{X}}, T_{\mathrm{XI}}, T_{\mathrm{XII}}, T_{\mathrm{XIII}}, T_{\mathrm{XIV}}\right.$, $\left.T_{\mathrm{XVI}}\right)$ are analogs of the sextet of $\mathrm{U}$ orbitals. They are also split into three triplets $\left(T_{\mathrm{X}}, T_{\mathrm{XI}}, T_{\mathrm{XIII}}\right.$ are localized on a one uranyl bond; $T_{\mathrm{XII}}, T_{\mathrm{XIV}}, T_{\mathrm{XVI}}$, on the other) and encompass each of these bonds from three sides. In contrast with the uranyl case, the examined trioxide LMOs are not fully equivalent because the uranyl group is not axially symmetric. All aforementioned types of active $\mathrm{U}$ subshells with $m_{l}=0, \pm 1, \pm 2, \pm 3$ in addition to $2 s-(6 \%)$ and $p_{x^{-}}, p_{y^{-}}$, and $p_{z^{-}}$-orbitals $(75 \%)$ of the corresponding $\mathrm{O}$ atom contribute to these LMOs. The U AOs that contribute most to the $T_{\mathrm{XII}}$ orbital (Fig. $3 \mathrm{c}$ ) are the $6 p_{x^{-}}(3 \%), 6 d_{+2^{-}}(1 \%)$, $6 d_{-2^{-}}(2 \%), 5 f_{+1^{-}}(2 \%), 5 f_{+3^{-}}(2 \%)$, and $5 f_{-3^{-}}$-orbitals $(3 \%)$. The next three LMOs are $\pi$-type $\left(T_{\mathrm{IX}}, T_{\mathrm{XVII}}, T_{\mathrm{XVIII}}\right)$ and are localized on the bond to the third $\mathrm{O}$ atom. They also encompass this bond from three sides and are not fully equivalent because of the presence of an $x z$ symmetry plane for the trioxide. Like for the $\pi$-type LMOs of the uranyl bonds, all aforementioned types of active $\mathrm{U}$ subshells with $m_{l}=0, \pm 1, \pm 2, \pm 3$ in addition to $2 s-(16 \%)$ and $p_{x^{-}}, p_{y^{-}}, p_{z^{-}}$-orbitals $(65 \%)$ of the third $\mathrm{O}$ atom contribute to this type of LMO. The U AOs that contribute most to the $T_{\mathrm{XVIII}}-\mathrm{orbital}$ (Fig. $3 \mathrm{~d}$ ) are the $6 p_{z^{-}}(6 \%), 6 d_{-2^{-}}(2 \%)$, $5 f_{0^{-}}(2 \%), 5 f_{+1^{-}}(1 \%)$, and $5 f_{-1^{-}}$orbitals $(4 \%)$. The remaining LMO $T_{\mathrm{XIX}}$ is also localized on the bond to the third $\mathrm{O}$ atom. However, it has a different shape due mainly to the $\mathrm{U} 6 p_{z^{-}}(53 \%), 6 d_{0^{-}}(3 \%)$, and $5 f_{0^{-}}$-orbitals $(6 \%)$ in addition to the $2 s$ $(11 \%)$ and $p_{z}$-orbitals $(21 \%)$ of the third $\mathrm{O}$ atom. As a result, LMO $T_{\mathrm{XIX}}$ is $\sigma$-type (Fig. 3e) and like $T_{\mathrm{VI}}$ has a lobe localized between the uranyl bonds.

For the Y-shape of the trioxide, LMOs $Y_{\mathrm{I}}, Y_{\mathrm{II}}, Y_{\mathrm{III}}, Y_{\mathrm{IV}}, Y_{\mathrm{VII}}, Y_{\mathrm{VIII}}$, and $Y_{\mathrm{XVIII}}$ are analogs of $T_{\mathrm{III}}, T_{\mathrm{II}}, T_{\mathrm{I}}, T_{\mathrm{IV}}, T_{\mathrm{VII}}$, $T_{\mathrm{VIII}}$, and $T_{\mathrm{XV}}$, respectively, of the T-shape LMOs and are not further examined. The remaining $12 \mathrm{LMOs}$ can be divided into six groups of one LMO $\left(Y_{\mathrm{V}}\right)$, one LMO $\left(Y_{\mathrm{VI}}\right)$, six LMOs $\left(Y_{\mathrm{IX}}, Y_{\mathrm{X}}, Y_{\mathrm{XIII}}, Y_{\mathrm{XVI}}, Y_{\mathrm{XVII}}, Y_{\mathrm{XIX}}\right)$, two LMOs $\left(Y_{\mathrm{XI}}, Y_{\mathrm{XIV}}\right)$, one LMO $\left(Y_{\mathrm{XII}}\right)$, and one $\mathrm{LMO}\left(Y_{\mathrm{XV}}\right)$. LMO $Y_{\mathrm{V}}$ is an analog of $T_{\mathrm{VI}}$. It is also localized mainly on the bond to the third $\mathrm{O}$ atom. However, it does not have the lobe characteristic of $T_{\mathrm{VI}}$ (Fig. $\left.4 \mathrm{a}\right)$. This is due to the smaller contribution of the U $6 p_{z}$-orbital $(60 \%)$ and more substantial contributions of the uranyl $\mathrm{O} s$ - and $p_{z}$-orbitals (14\%) while preserving the previous contribution of the $s$ - and $p_{z}$-orbitals of the third $\mathrm{O}$ atom (24\%). LMO $Y_{\mathrm{VI}}$ (Fig. $\left.4 \mathrm{~b}\right)$ is an analog of $T_{\mathrm{V}}$. It is formed from U $6 p_{x}$-orbitals $(66 \%)$ in addition to uranyl $\mathrm{O} s-(30 \%)$ and $p_{x}$-orbitals $(1 \%)$. The total contribution of $\mathrm{U}$ and $\mathrm{O}$ orbitals to non-zero $z$-projections is $\sim 3 \%$. The next six LMOs of the Y-shape ( $\left.Y_{\mathrm{IX}}, Y_{\mathrm{X}}, Y_{\mathrm{XIII}}, Y_{\mathrm{XVI}}, Y_{\mathrm{XVII}}, Y_{\mathrm{XIX}}\right)$ are obviously analogs of the corresponding sextet of T-shape orbitals and have similar characteristics. LMOs $Y_{\mathrm{IX}}, Y_{\mathrm{XIII}}$, and $Y_{\mathrm{XVII}}$ are localized on one uranyl bond; $Y_{\mathrm{X}}, Y_{\mathrm{XVI}}$, and $Y_{\mathrm{XIX}}$, on the other. Like for the T-shape, all aforementioned types of active $\mathrm{U}$ subshells with $m_{l}=0, \pm 1, \pm 2, \pm 3$ in addition to $2 s^{-}(8 \%)$ and $p_{x^{-}}, p_{y^{-}}, p_{z^{-}}$-orbitals $(70 \%)$ of the corresponding $\mathrm{O}$ atoms contribute to such LMOs. The $\mathrm{U}$ AOs $6 p_{x^{-}}(4 \%)$, $6 p_{z^{-}}(4 \%), 6 d_{0^{-}}(2 \%), 6 d_{-1^{-}}(1 \%), 6 d_{-2^{-}}(1 \%), 5 f_{0^{-}}(1 \%), 5 f_{+1^{-}}(3 \%), 5 f_{+2^{-}}(1 \%), 5 f_{-2^{-}}(2 \%), 5 f_{-3^{-}}$-orbitals $(1 \%)$ contribute most to the $Y_{\mathrm{XVII}}$-orbital (Fig. 4f). The pair of LMOs $Y_{\mathrm{XI}}$ and $Y_{\mathrm{XIV}}$ and another LMO $Y_{\mathrm{XII}}$ of the Y-shape are analogs of 

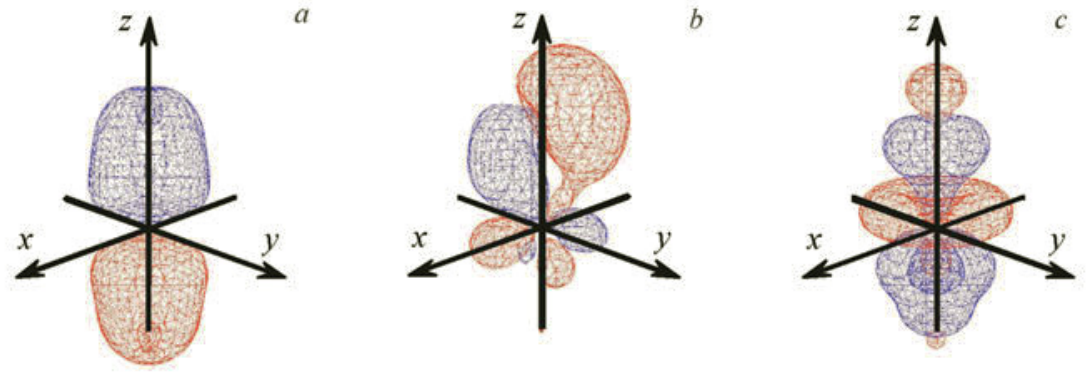

Fig. 2. Localized orbitals of uranyl ion: $U_{\mathrm{IV}}(\mathrm{a}), U_{\mathrm{VII}}(\mathrm{b})$, and $U_{\mathrm{XIV}}(\mathrm{c})$.
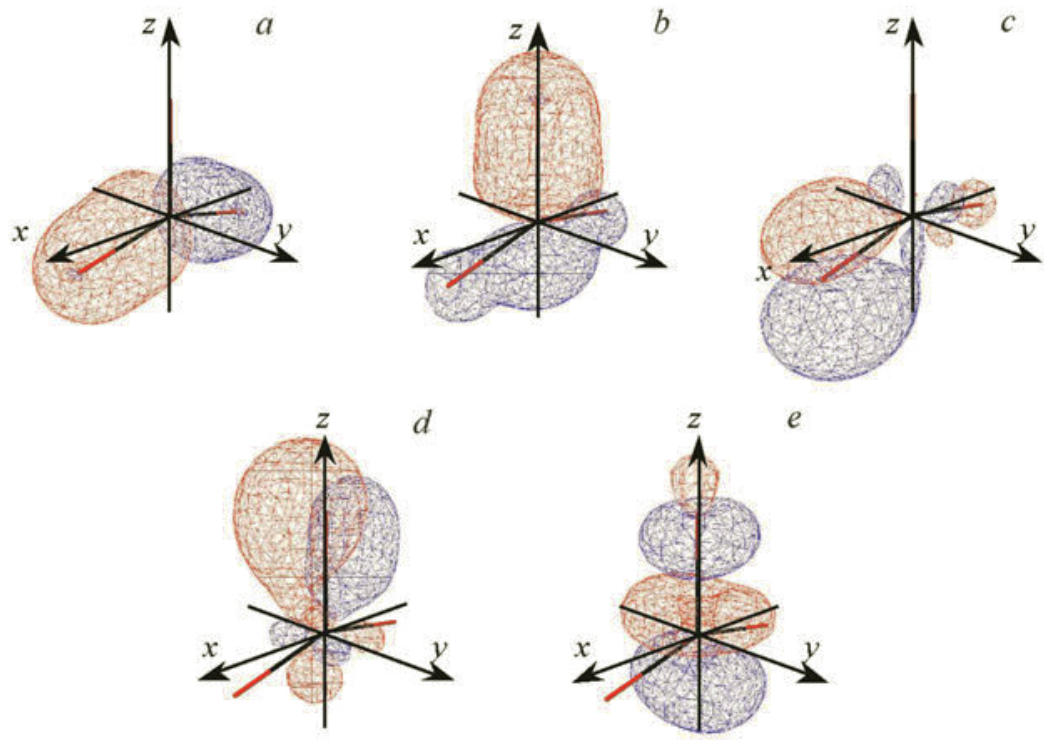

Fig. 3. Localized orbitals of the T-shape of $\mathrm{UO}_{3}: T_{\mathrm{V}}(\mathrm{a}), T_{\mathrm{VI}}(\mathrm{b}), T_{\mathrm{XVI}}(\mathrm{c}), T_{\mathrm{XVIII}}(\mathrm{d})$, and $T_{\mathrm{XIX}}(\mathrm{e})$.
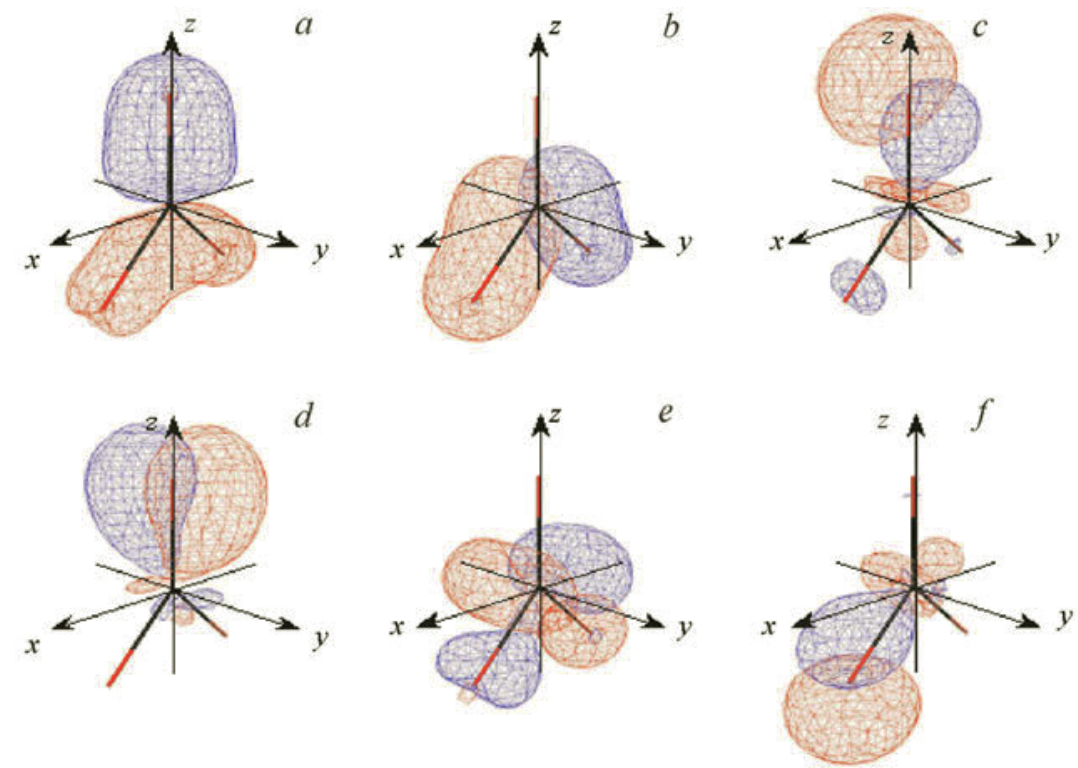

Fig. 4. Localized orbitals of the Y-shape of $\mathrm{UO}_{3}: Y_{\mathrm{V}}(\mathrm{a}), Y_{\mathrm{VI}}(\mathrm{b}), Y_{\mathrm{XI}}(\mathrm{c}), Y_{\mathrm{XII}}(\mathrm{d}), Y_{\mathrm{XV}}(\mathrm{e})$, and $Y_{\mathrm{XVII}}(\mathrm{f})$. 
three $\pi$-type LMOs of the T-shape that are localized on the bond to the third $\mathrm{O}$ atom ( $T_{\mathrm{IX}}, T_{\mathrm{XVII}}$, and $\left.T_{\mathrm{XVIII}}\right)$. LMO $Y_{\mathrm{XII}}$ (Fig. 4d), in contrast with $T_{\mathrm{XVIII}}$ (Fig. 3d), is symmetric relative to the $x z$ plane and; therefore, is placed into a separate group. All aforementioned types of active $\mathrm{U}$ subshells with $m_{l}=0, \pm 1, \pm 2, \pm 3$ in addition to $2 s$ - (8\%) and $p_{x^{-}}$and $p_{z}$-orbitals $(67 \%)$ of the third $\mathrm{O}$ atom contribute to the $Y_{\mathrm{XI}}$ and $Y_{\mathrm{XIV}}$ LMOs. The U AOs $7 s^{-}(2 \%), 6 p_{z^{-}}(3 \%), 6 d_{0^{-}}(2 \%), 6 d_{+1^{-}}(2 \%), 5 f_{0^{-}}(6 \%)$, and $5 f_{+1}$-orbitals (4\%) contribute most to the $Y_{\mathrm{XI}}$-orbital (Fig. 4c). The structure of the $Y_{\mathrm{XII}} \mathrm{LMO}$ is simpler. It is formed from $\mathrm{U} 6 d_{-1^{-}}(4 \%)$ and $5 f_{-1^{-}}$-orbitals $(7 \%)$ and $p_{y^{-}}$-orbitals $(88 \%)$ of the third $\mathrm{O}$ atom. The remaining LMO of the Y-shape, $Y_{\mathrm{XV}}$, has no analog among the T-shape LMOs and is formed from $\mathrm{U}$ and uranyl O AOs with non-zero $x$-projections, i.e., $6 p_{x^{-}}(60 \%)$, $6 d_{+1^{-}}(3 \%), 5 f_{+1^{-}}(7 \%)$, and $5 f_{+3^{-}}$-orbitals $(1 \%)$ of the $\mathrm{U}$ atom and $2 s-(15 \%)$ and $p_{x^{-}}$and $p_{z^{-}}$-orbitals $(14 \%)$ of the uranyl $\mathrm{O}$ atoms. A characteristic feature of the $Y_{\mathrm{XV}}$-orbital (Fig. 4e) is the presence of two lobes localized at corners formed by the uranyl bonds and the bond to the third $\mathrm{O}$ atom.

Thus, in our opinion, important factors for stabilizing the T-shape of the trioxide are the substantial contributions of the $6 p_{z}$-orbital to $\sigma$-type LMOs $T_{\mathrm{VI}}$ and $T_{\mathrm{XIX}}$ localized on the bond to the third $\mathrm{O}$ atom and forming lobes situated between the uranyl bonds and thereby repelling them from each other. In turn, the dominant contribution of the $6 p_{x}$-orbital to LMO $Y_{\mathrm{XV}}$ that is responsible for forming the lobes that press the uranyl bonds toward each other can stabilize the Y-shape of the trioxide.

Conclusions. Quantum chemical modeling in terms of density functional theory LANL2DZ/B3LYP/cc-pVDZ predicted the existence of two stable conformers of molecular $\mathrm{UO}_{3}$. We attempted to explain this phenomenon on the basis of concepts of LMOs because their shape and structure reflect features of the electronic and geometric structures of molecular systems. Analytical results for LMOs of uranyl and the two shapes of $\mathrm{UO}_{3}$ indicated that $\mathrm{U}$ AOs contribute most to the $\sigma$-type bonds; the O AOs, to the $\pi$-type. As a result, $\pi$-type LMOs in the trioxide preserve mainly the structure and shape of the corresponding $\mathrm{U}$ orbitals. The principal differences in LMOs of the T- and Y-shapes of $\mathrm{UO}_{3}$ are related to the different contributions of $\mathrm{U} A O s$ and those of the third $\mathrm{O}$ atom in the molecular plane for the Y-shape and the lack of such localization for the T-shape. These differences of LMOs are reflected directly in the structures of the two shapes of $\mathrm{UO}_{3}$.

The existence of the examined conformers is hypothetical in nature. Experimental confirmation of the stability of the Y- and T-shapes of $\mathrm{UO}_{3}$ would be a unique example of a bistable molecular system with the bond angle as the coordinates of large-amplitude intramolecular motion.

\section{REFERENCES}

1. P. V. R. Schleyer, E. U. Wüerthwein, E. Kaufmann, T. Clark, and J. A. Pople, J. Am. Chem. Soc., 105, 5930-5932 (1983).

2. H. Kudo, Nature, 355, 432-434 (1992).

3. W. Zhizhong, Z. Xiange, and T. Auchin, J. Mol. Struct.: THEOCHEM, 453, 225-231 (1998).

4. Z.-W. Fu, L.-N. Zhang, Q.-Z. Qiu, Y.-H. Zhang, X.-K. Zeng, H. Cheng, R.-B. Huang, and L.-S. Zheng, J. Phys. Chem. A, 104, 2980-2984 (2000).

5. L. Andrews, B. Liang, J. Li, and B. E. Bursten, J. Am. Chem. Soc., 125, 3126-3139 (2003).

6. L. Gagliardi and B. O. Roos, Nature, 433, 848-851 (2005).

7. S. D. Gabelnick, G. T. Reedy, and M. G. Chasanov, J. Chem. Phys., 58, $4468-4475$ (1973).

8. S. D. Gabelnick, G. T. Reedy, and M. G. Chasanov, J. Chem. Phys., 59, 6397-6404 (1973).

9. D. W. Green, G. T. Reedy, and S. D. Gabelnick, J. Chem. Phys., 73, 4207-4216 (1980).

10. R. D. Hunt and L. Andrews, J. Chem. Phys., 98, 3690-3696 (1993).

11. M. Zhou, L. Andrews, N. Ismail, and C. Marsden, J. Phys. Chem. A, 104, 5495-5502 (2000).

12. P. Li, T.-T. Jia, T. Gao, and G. Li, Chin. Phys. B, 21, 043301 (2012).

13. R. G. Denning, J. Phys. Chem. A, 111, 4125-4143 (2007).

14. M. B. Shundalau, A. P. Zajogin, A. I. Komiak, A. A. Sokolsky, and D. S. Umreiko, J. Spectrosc. Dyn., 2, 19 (2012).

15. O. Chalvet, R. Daudel, S. Diner, and J. P. Malrieu, Localization and Delocalization in Quantum Chemistry, Dordrecht, Holland (1975).

16. M. W. Schmidt, K. K. Baldridge, J. A. Boatz, S. T. Elbert, M. S. Gordon, J. H. Jensen, S. Koseki, N. Matsunaga, K. A. Nguyen, S. J. Su, T. L. Windus, M. Dupuis, and J. A. Montgomery, J. Comput. Chem., 14, 1347-1363 (1993).

17. http://www.msg.ameslab.gov/GAMESS/GAMESS.html

18. B. M. Bode and M. S. Gordon, J. Mol. Graphics Modell., 16, 133-138 (1998).

19. L. J. Farrugia, J. Appl. Crystallogr., 30, 565 (1997).

20. P. J. Hay and R. L. Martin, J. Chem. Phys., 109, 3875-3881 (1998). 
21. T. H. Dunning, Jr., J. Chem. Phys., 90, 1007-1023 (1989).

22. https://bse.pnl.gov/bse/portal

23. D. Feller, J. Comput. Chem., 17, 1571-1586 (1996).

24. K. L. Schuchardt, B. T. Didier, T. Elsethagen, L. Sun, V. Gurumoorthi, J. Chase, J. Li, and T. L. Windus, J. Chem. Inf. Model., 47, 1045-1052 (2007).

25. A. D. Becke, J. Chem. Phys., 98, 5648-5652 (1993).

26. C. Lee, W. Yang, and R. G. Parr, Phys. Rev. B: Condens. Matter Mater. Phys., 37, 785-789 (1988).

27. P. J. Stephens, F. J. Devlin, C. F. Chabalowski, and M. J. Frisch, J. Phys. Chem., 98, 11623-11627 (1994).

28. M. B. Shundalau, A. I. Komyak, A. P. Zazhogin, and D. S. Umreiko, Zh. Prikl. Spektrosk., 79, 27-36 (2012).

29. M. B. Shundalau, A. I. Komiak, A. P. Zajogin, and D. S. Umreiko, J. Spectrosc. Dyn., 3, 4 (2013).

30. C. Edmiston and K. Ruedenberg, Rev. Mod. Phys., 35, 457-464 (1963).

31. P. Pyykkö, J. Li, and N. Runeberg, J. Phys. Chem., 98, 4809-4813 (1994).

32. J. C. Eisenstein and M. H. L. Pryce, Proc. R. Soc. London, Ser. A, 229, 20-38 (1955).

33. S. P. McGlynn and J. K. Smith, J. Mol. Spectrosc., 6, 164-187 (1961).

34. P. Pyykkö and L. L. Lohr, Jr., Inorg. Chem., 20, 1950-1959 (1981).

35. G. Schreckenbach, P. J. Hay, and R. L. Martin, Inorg. Chem., 37, 4442-4452 (1998).

36. N. Ismail, J.-L. Heully, T. Saue, J.-P. Daudey, and C. J. Marsden, Chem. Phys. Lett., 300, 296-302 (1999).

37. K. G. Dyall, Mol. Phys., 96, 511-518 (1999).

38. W. A. de Jong, R. J. Harrison, J. A. Nichols, and D. A. Dixon, Theor. Chem. Acc., 107, 22-26 (2001). 